\title{
Diagnóstico de situação de processos de desinfecção e esterilização de materiais dos estabelecimentos de beleza com serviços de manicure e pedicura no município de Garanhuns
}

\author{
[Situation diagnosis of processes of disinfection and sterilization of materials of beauty \\ establishments with manicure and pedicure services in the municipality of Garanhuns]
}

\section{"Artigo Científico/Scientific Article"}

\section{Cleber Vinicius Brito dos Santos ${ }^{1 *}$, Luana Cardoso da Silva², Maria Elisa de Almeida Araújo ${ }^{3}$, Daniel Friguglietti Brandespim ${ }^{1}$}

\author{
${ }^{1}$ Departamento de Medicina Veterinária, Universidade Federal Rural de Pernambuco, Recife-PE, Brasil. \\ ${ }^{2}$ Unidade Acadêmica de Garanhuns (UAG), Universidade Federal Rural de Pernambuco, Garanhuns -PE, Brasil. \\ ${ }^{3}$ Secretaria Municipal de Saúde da Prefeitura da Cidade do Recife, Recife-PE, Brasil. \\ *Autor para correspondência/Corresponding author: E-mail: cleber.vini@yahoo.com.br
}

\section{Resumo}

O estudo objetivou descrever o perfil de estabelecimentos de beleza, no tocante a presença de alvará sanitário e conhecimento dos profissionais em relação ao risco de transmissão de patógenos e legislação sanitária no município de Garanhuns-PE. A coleta de dados foi feita por meio de entrevistas e aplicação de questionário semiestruturado e anônimo. Dentre os 53 estabelecimentos participantes, apenas $3(5,7 \%)$ funcionaram com a licença sanitária, sendo que a maioria dos estabelecimentos $(62,3 \%)$ não utilizavam qualquer processo de higienização de equipamentos, mesmo com $54,7 \%$ deles sabendo da possibilidade de transmissão de doenças no exercício das atividades. É de fundamental importância a conscientização dos proprietários da necessidade do alvará sanitário para funcionamento do estabelecimento, além de ações educacionais pelos órgãos de fiscalização, para adequação às práticas de correta esterilização dos materiais utilizados e informação sobre as doenças passíveis de transmissão entre cliente e profissional e vice-versa, para garantir a segurança nos estabelecimentos prestadores de serviços, que se relacionam diretamente com a saúde da população.

Palavras-Chave: Vigilância sanitária; salões de beleza; promoção em saúde; prevenção em saúde.

\begin{abstract}
This study aims to describe the profile of beauty shops, concerning the presence of sanitary and business licenses and knowledge of the professionals regarding the risk of transmission of pathogens and sanitary legislation in the municipality of Garanhuns-PE. Data collection was made via interviews and the use of a semi-structured and anonymous questionnaire. Among the participating establishments $(n=53)$, only $3(5.7 \%)$ worked with a sanitary license, and the majority of the establishments $(62.3 \%)$ did not use any sterilization process, even though $54.7 \%$ of them recognized the possibility of disease transmission during their activities. It is of fundamental importance that the owners be aware of the necessity of a sanitary permit for the operation of the establishment, followed by educational efforts by oversight agencies, to adopt practices of correct sterilization of the materials used and information about the diseases that may be transmitted between client and professional, and vice versa, to ensure safety for the service providers, which is directly related to the health of the population.
\end{abstract}

Keywords: Health surveillance; beauty salon; health promotion; disease prevention.

\section{Introdução}

Diariamente, milhares de brasileiros visitam salões de beleza em busca de cuidados estéticos e que lhes proporcione bem-estar. Os salões de beleza se transformaram em locais de encontro social, onde são feitos procedimentos estéticos que Recebido em: 23 de março de 2017.

Aceito em: 29 de outubro de 2017. podem vir a gerar risco de transmissão de doenças e agravos (Kiec-Swierczynska et al., 2013).

A lei orgânica da saúde (Lei no 8080/90), que regula o Sistema Único de Saúde (SUS), estabelece a descentralização das atividades de saúde para estados e municípios (BRASIL, 1990). Dessa 
forma, o Sistema Nacional de Vigilância Sanitária, que integra o SUS, opera de forma integrada e descentralizada em todo o país, coordenado pela Agência Nacional de Vigilância Sanitária (ANVISA), que tem por funções, executar e regulamentar as ações de abrangência nacional, cabendo aos municípios e estados as ações de fiscalização e regulamentação complementares.

Os salões de beleza enquadram-se nos serviços de interesse à saúde e são considerados serviços de baixa complexidade, mesmo com os riscos eminentes a essas atividades. Na realização dos procedimentos estéticos, podem ocorrer lesões na pele e anexos e outros agravos (KiecSwierczynska et al., 2013).

$\mathrm{O}$ instrumental e outros materiais utilizados em salões de beleza constituem uma importante via de transmissão de microrganismos caso não seja feito o descarte ou desinfecção de forma adequada após a utilização (Diniz e Matté, 2013)

$\mathrm{O}$ risco mais preocupante nos estabelecimentos de embelezamento é a possibilidade de se contrair doenças infecciosas, se destacando a AIDS (transmitida pelo HIV), a Hepatite B (transmitida pelo HBV) e a hepatite C (transmitida pelo HCV) (Silva et al., 2014), tornando-se iminente o risco quando, principalmente, manicures e pedicuras desconhecem ou não seguem as normas de biossegurança, como a utilização de Equipamentos de Proteção Individual (EPIs), adequado processamento dos instrumentos, correto descarte de materiais descartáveis e higienização (de mãos e ambiente), como destacado por Garbaccio e Oliveira (2013).

As atividades realizadas nos salões de beleza favorecem a transmissão cruzada de microrganismos entre profissionais e clientes, especialmente quando ocorrem acidentes com materiais perfuro-cortantes e com exposição sanguínea (Kiec-Swierczynska et al., 2013). No estudo de Diniz e Matté (2013), foi constatado que $45 \% \quad(n=18)$ dos profissionais tiveram contato com sangue do cliente sem estar usando luvas, enquanto Oliveira e Focaccia (2010) observaram que $8 \% \quad(n=8)$ de manicures e pedicuras em salões de beleza tiveram contato com o vírus da hepatite B e $2 \%(n=2)$ com o vírus da hepatite $\mathrm{C}$ e, além disso, a adesão a normas de biossegurança observada foi inadequada e baixa.
Dessa forma, os estabelecimentos de beleza devem respeitar e adequar-se às normas sanitárias vigentes, com a finalidade de garantir segurança tanto a profissionais quanto à população que busca pelos serviços (Garbaccio e Oliveira, 2013).

Nesse contexto, o presente artigo objetivou descrever o perfil de estabelecimentos de beleza em Garanhuns-PE, no tocante a presença de alvará sanitário e conhecimento dos profissionais em relação ao risco de transmissão de patógenos e legislação sanitária estabelecida pela ANVISA.

\section{Material e Métodos}

Tipo de estudo e público alvo

Foi realizado um estudo do tipo descritivo, no qual os sujeitos de pesquisa foram os representantes de estabelecimentos que fornecem algum tipo de serviço de beleza, no município de Garanhuns, Pernambuco, Brasil.

Fonte dos dados

Os dados foram obtidos por meio de entrevistas e aplicação de questionário semiestruturado, sem identificação dos sujeitos, dividido em 3 blocos temáticos, com as seguintes variáveis: bloco I - Questões referentes ao estabelecimento e seu funcionamento (serviços oferecidos no estabelecimento; posse de licença sanitária; conhecimento da documentação necessária para licenciamento e conhecimento sobre a existência e importância da ANVISA), bloco II - Questões referentes ao manejo higiênico dos produtos e materiais utilizados (esterilização dos instrumentais e métodos utilizados) e bloco III - Questão referente ao conhecimento de doenças passíveis de transmissão no ambiente de trabalho.

A coleta de dados foi realizada entre os meses de setembro e dezembro de 2014, com contato prévio por telefone para agendamento da visita. A partir desses dados, foi criado um banco de dados no Excel, que posteriormente foi exportado para análise estatística no Statistical Package for Social Sciences Version 23 (IBM CORP., 2015).

\section{Preceitos éticos}

Previamente à coleta, todos participantes foram orientados quanto aos objetivos do estudo, seu anonimato e do estabelecimento e, quando de acordo, os participantes foram convidados a assinar o termo de consentimento livre e esclarecido (TCLE), respeitando-se os preceitos éticos da Resolução do Conselho Nacional de Saúde (CNS) 
$\mathrm{n}^{\circ}$ 466, de 12 de dezembro de 2012, considerando o respeito pela dignidade humana, anonimato de participantes, o direito de desistir em participar da pesquisa a qualquer momento, além das orientações do consentimento livre e esclarecido dos sujeitos.

\section{Resultados}

No total, 53 representantes de estabelecimentos aceitaram participar do estudo, onde foram verificados vários tipos de serviços prestados, sendo predominantes os estabelecimentos que ofereciam exclusivamente serviços de cabeleireiro (41,5\%), os dados detalhados sobre os tipos de serviços podem ser observados na Tabela 1.

Tabela 1. Tipos de serviços prestados nos estabelecimentos de beleza, Garanhuns-PE, de setembro a dezembro de 2014.

\begin{tabular}{|c|c|c|}
\hline Serviços Oferecidos & $\mathbf{N}^{\mathbf{o}}$ & $\%$ \\
\hline Cabeleireiro & 22 & 41,5 \\
\hline Cabeleireiro + Manicure/pedicura & 11 & 20,7 \\
\hline Barbearias & 10 & 18,8 \\
\hline Manicure / Pedicura & 5 & 9,5 \\
\hline Cabeleireiro + Maquiagem & 1 & 1,9 \\
\hline Cabeleireiro + Manic/pedic ${ }^{1}+$ Maqui $^{2}$ & 1 & 1,9 \\
\hline Cabeleireiro + Depil ${ }^{3}+$ Manic/pedic ${ }^{1}$ & 1 & 1,9 \\
\hline Cabeleireiro + Depil $^{3}+$ Maqui $^{2}+$ Manic/pedic $^{1}$ & 1 & 1,9 \\
\hline Manicure/Pedicura + Depilação & 1 & 1,9 \\
\hline Total & 53 & $\mathbf{1 0 0 , 0}$ \\
\hline
\end{tabular}

${ }^{1}$ Manicure e/ou pedicura; 2 Maquiagem; 3 Depilação

Dos 53 estabelecimentos participantes, apenas $3(5,7 \%)$ dos entrevistados afirmaram que funcionam com a licença sanitária e, entre os não licenciados, $42(84,0 \%)$ disseram não ter conhecimento sobre a documentação necessária para a obtenção da licença, apesar de apenas 12 entrevistados $(22,7 \%)$ relatarem não ter conhecimento sobre o que é a ANVISA e sua importância (Tabela 2).

Tabela 2. Características referentes a porte de licença sanitária, documentação necessária para a licença e conhecimento da ANVISA, Garanhuns-PE, de setembro a dezembro de 2014.

\begin{tabular}{lcc}
\hline & $\mathbf{N}^{\mathbf{0}}$ & $\%$ \\
\hline Licença sanitária & 50 & 94,3 \\
Não & 3 & 5,7 \\
Sim & & 84,0 \\
Documentos necessários & 42 & 16,0 \\
Não & 8 & 77,3 \\
Sim & & 22,7 \\
Conhecimento da $\boldsymbol{A N V I S A}$ & 41 & \\
Sim & 12 & \\
Não & & \\
\hline
\end{tabular}

De acordo com os dados da Tabela 3, em relação aos processos de higienização de equipamentos nos estabelecimentos, observou-se a utilização de estufa, álcool, água e sabão ou água quente como métodos de desinfecção dos equipamentos, sendo que a maioria dos estabelecimentos $(62,3 \%)$ não utilizavam qualquer processo de higienização de equipamentos, enquanto $24,5 \%$, utilizaram apenas a estufa, porém sem controle de temperatura e tempo no processo de desinfecção dos equipamentos.

Em relação ao conhecimento sobre a possibilidade de transmissão de doenças no exercício das atividades e sobre quais seriam tais doenças, a maioria $(54,71 \%)$ dos representantes conheciam o risco e, dentre eles, as doenças mais citadas foram as hepatites $(65,5 \%)$, seguida pelo HIV e fúngicas (Tabela 4). 
Tabela 3. Processos de desinfecção e limpeza utilizados de equipamentos nos estabelecimentos de beleza visitados no município de Garanhuns no período de setembro a dezembro de 2014.

\begin{tabular}{lcc}
\hline \multicolumn{1}{c}{ Processo de limpeza } & $\mathbf{N}^{\mathbf{0}}$ & $\mathbf{\%}$ \\
\hline Estufa & 13 & 24,5 \\
Estufa + álcool & 3 & 5,66 \\
Álcool & 2 & 3,8 \\
Água e sabão & 1 & 1,9 \\
Água Quente & 1 & 1,9 \\
Não o faz & 33 & 62,3 \\
\hline \multicolumn{1}{c}{ Total } & $\mathbf{5 3}$ & $\mathbf{1 0 0 , 0}$ \\
\hline
\end{tabular}

Tabela 4. Número e percentual de respostas sobre o conhecimento de doenças transmitidas por materiais não esterilizados em estabelecimentos de beleza visitados no município de Garanhuns, no período de setembro a dezembro de 2014.

\begin{tabular}{lcc}
\hline & $\mathbf{N}^{\mathbf{0}}$ & $\mathbf{\%}$ \\
\hline Transmissão de doenças & & 54,7 \\
Sim & 29 & 45,3 \\
Não & 24 & \\
Doenças & & 65,5 \\
Hepatites (B e C) & 19 & 27,5 \\
HIV & 8 & 7,0 \\
Fungos & 2 & $\mathbf{1 0 0 , 0}$ \\
\hline Total & $\mathbf{2 9}$ & \\
\hline
\end{tabular}

\section{Discussão}

As ações de vigilância na proteção e promoção da saúde no que se refere a salões de beleza são coordenadas e implantadas pela ANVISA, que disponibiliza materiais de referência técnica para o correto funcionamento dos serviços de estética e embelezamento (BRASIL, 2009), sendo de competência da ANVISA o papel de desenvolver ações para prevenir ou diminuir os riscos a saúde e intervir em problemas sanitários decorrente desse tipo de serviço no país (BRASIL, 1990).

Porém, para tais ações serem implementadas e cumpridas pelos estabelecimentos, estes necessitam de alvará sanitário, ou licença sanitária, que deverá ser autorizado pelos órgãos de vigilância sanitária municipais, permitindo à ANVISA uma atuação mais sistematizada das suas ações.

Os resultados dessa pesquisa demonstraram que a maioria $(62,3 \%)$ dos 33 estabelecimentos não utilizou nenhum método para controle de risco biológico e dentre os que utilizam algum método, 24,5\% ( $\mathrm{n}=13)$ utilizaram o esterilizador simples (estufa), ressaltando-se que a ANVISA recomenda que equipamentos devem ser lavados com água, detergente e escova de cerdas macias e, após limpos, devem ser embalados e esterilizados em autoclaves (BRASIL, 2009), procedimento este não observado em nenhum dos estabelecimentos visitados.

Para o material ser considerado esterilizado, se faz necessário manter a integridade e qualidade das embalagens, dessa maneira o invólucro forma uma barreira de isolamento, e quanto a efetividade do produto ter sido realmente esterilizado, deve-se confirmar se os indicadores biológicos e químicos estão sendo respeitados, de forma a evitar o crescimento microbiano nos objetos (Maldaner et al., 2013).

Ao contrário dos estudos de Garbaccio e Oliveira (2013) e Oliveira e Focaccia (2010), onde a maioria dos profissionais dizia desconhecer a possibilidade de transmissão de doenças por meio do instrumental de trabalho, nossos resultados demonstraram que a maioria dos entrevistados $(n=29)$ teve ciência do risco, tanto para eles, quanto para os clientes, da transmissão de doenças. Entre os que afirmavam saber do risco de transmissão, as doenças mais citadas foram as hepatites, corroborando com a afirmação de Garbaccio e Oliveira (2013), que demonstraram as hepatites como doenças de maior ocorrência em salões de beleza. 
É de extrema importância que os profissionais deste segmento tenham formação e capacitação. Tais ações resultam no oferecimento de um serviço seguro, diminuindo os riscos de agravos à saúde, que são minimizados pela fiscalização dos estabelecimentos. Porém, para isso ocorrer, é necessária a licença sanitária dos estabelecimentos (Schwaab et al., 2015; Silva et al., 2007). Dessa forma, os estabelecimentos devem ser objetos de fortes ações educativas sobre as medidas preventivas (Melo e Isolani, 2011).

\section{Conclusão}

É de grande importância a conscientização dos proprietários dos estabelecimentos de beleza da necessidade do alvará sanitário para funcionamento do estabelecimento, além de planejamento de ações educacionais para o adequado funcionamento desses estabelecimentos, adequando-se a práticas de higienização dos materiais, além da informação sobre as doenças passíveis de transmissão, visando garantir a saúde da população.

\section{Conflito de Interesse}

Os autores declaram não existir conflito de interesse.

\section{Referências}

BRASIL. Agência Nacional de Vigilância Sanitária. Referência técnica para o funcionamento dos serviços de estética e embelezamento sem responsabilidade médica. Brasília (DF). 2009. Disponível em:http://portal.anvisa.gov.br/wps/wcm/c onnect/527126804745890192e5d63fbc4c $6735 /$ Servicos+de+Estetica+e+Congenere s.pdf?MOD=AJPERES. Acesso em: $07 \mathrm{fev}$ 2017.___. Lei no 8080, de 19 de setembro de 1990. Dispõe sobre as condições para a promoção, proteção e recuperação da saúde, a organização e o funcionamento dos serviços correspondentes e dá outras providências. Diário Oficial da União. Seção 1:018055, 1990.
Diniz, A.F.; Matté, G.R. Procedimentos de biossegurança adotados por profissionais de serviços de embelezamento. Saúde e Sociedade, 22(3):751-759, 2013.

Garbaccio J.L.; Oliveira A.C. O risco oculto no segmento de estética e beleza: Uma avaliação do conhecimento dos profissionais e das práticas de biossegurança nos salões de beleza. Texto contexto enfermagem, 22(4): 989-98, 2013

IBM Corp -International Business Machines Corp. IBM SPSS for Macintosh. Version 23.0. Armonk (NY): IBM Corp.; 2015.

Kieć-Swierczyńska, M.; Chomiczewska-Skóra, D.; Swierczyńska-Machura, D.; Krecisz, B. Manicurists and pedicurists occupation group at high risk of workrelated dermatoses. Medycyna Pracy, 64(4): 579-91, 2013.

Maldaner, C.; Berlet, L.J.; Ascari, R.A.; Klein, M.L.; Savian, B.A.; Silva, O.M.D. Invólucros para esterilização de materiais odonto-médico-hospitalares. Revista de Saúde Pública de Santa Catarina, 6(3): 61-70, 2013

Melo, F.C.A.D.; Isolani, A.P. Hepatite B e C: do risco de contaminação por materiais de manicure/pedicura à prevenção. SaBios: Revista de Saúde e Biologia, 6(2): 72-78 2011.

Oliveira A.C.D.S.; Focaccia R. Survey of hepatitis $\mathrm{B}$ and $\mathrm{C}$ infection control: procedures at manicure and pedicure facilities in São Paulo. Brazilian Journal of Infectious Diseases, 14(5): 502-507, 2010.

Schwaab, G.; Lunkes, J. T.; Jacob, A. M.; Fermino, N.; Silva, O. M.; Ascari, R. A. Reprocessamento de materiais em estabelecimentos de beleza. Revista UNINGÁ, 43: 44-49, 2015.

Silva, L.F.M.; Albuquerque, C.; Ferreira, L. Informações sobre as práticas do processo de esterilização dos tatuadores e "piercers" do Recife-PE 2006. Revista de Vigilância Sanitária, 3(1/2/3): 313-319, 2007.

Silva, L.M.S.; Silva, D.C.; Diniz, J.S.; Felipe Ima, N.S.P.H. Prevenção da transmissão de hepatites virais entre manicures e pedicuras uma revisão. Infarma-Ciências Farmacêuticas, 26(2): 82-89, 2014. 